\title{
Humor Modeling in the Interface
}

\author{
Anton Nijholt \\ University of Twente \\ Enschede, Netherlands \\ anijholt@cs.utwente.nl
}

\author{
Oliviero Stock \\ ITC-IRST \\ Trento, Italy \\ stock@itc.it
}

\author{
Alan Dix \\ Lancaster University \\ Lancaster, UK \\ alan@hcibook.com
}

\author{
John Morkes \\ Trilogy, Austin, \\ TX, USA \\ John_Morkes@trilogy.com
}

\section{INTRODUCTION}

Humor is a multi-disciplinary field of research. People have been working on humor in many fields of research, such as psychology, philosophy and linguistics, sociology and literature. Especially in the context of computer science (or Artificial Intelligence) humor research aims at modeling humor in a computationally tractable way. Having computational models of humor allows interface designers to have the computer generate and interpret humor when interacting with users. Being able to recognize a user's frustration [5] can be useful; however, preventing it whenever possible by an adequate use of humor can be useful as well.

There are many situations in human-human interaction where humor plays an important role. It does not only play an entertaining role. It helps in regulating conversations (social and business conversations), building trust between partners and facilitating self-disclosure and it is an important factor in social attraction.

Nass has shown in different studies [7, 10] that humans respond in the same way to computers as they do to persons with respect to psychosocial phenomena such as personality, politeness, flattery, and in-group favoritism. Making use of this paradigm we may investigate a similar role to be played in human-computer interaction for various types of humor use and we can see whether the regulating and social-psychological aspects of humor can play positive roles in human-computer interaction.

\section{HUMOR IN HUMAN-HUMAN INTERACTION}

In interpersonal interactions, either at work or at home, humans use humor, humans smile and humans laugh. Humor can be spontaneous, but it can also serve a social role and be used deliberately. A smile can be the effect of appreciating a humorous event, but it can also be used to regulate the conversation. A laugh can be spontaneous but can also mask disagreement or be cynical. Research has shown that laughs are related to topic shifts in a conversation and phases in negotiations or problem solving tasks.

For our aims we are not particularly interested in a possible preconceived aim of a conversational partner to create humor during a conversation or discussion. Rather we look at situations where humor occurs more or less spontaneously

Copyright is held by the author/owner(s).

CHI 2003, April 5-10, 2003, Ft. Lauderdale, Florida, USA.

ACM 1-58113-637-4/03/0004 during an interaction, where it occurs in a supporting role, for example to hide embarrassment, to dominate the discussion or to change the topic, or where it induces trust or has a role in interpersonal attraction [2]. Humor support, or the reaction to humor, is an important aspect of interpersonal interaction. Humans employ a wide range of humor in their conversations and the given support shows the understanding and appreciation of humor. There are many support strategies. The strategy that can be used in a certain situation is mainly determined by the context of the humorous event. The strategy can include smiles and laughter, the contribution of more humor, echoing the humor, offering sympathy or contradicting self-deprecating humor. There are also situations in which no support is necessary. In order to give full humor support, humor has to be recognized, understood and appreciated. These factors determine our level of agreement on a humorous event and the grade of how we want to support the humor. Support may show our involvement in the discussion, our motivation to continue and how much we enjoy the conversation or interaction.

\section{HUMOR IN HUMAN-COMPUTER INTERACTION}

It can be expected that in future applications, not only in specially designed entertainment environments, humancomputer interaction will become less goal-directed than in the past. Information and communication technology moves to the living room and all sorts of social environments where we have human- (embodied) agent interaction and computer-mediated interaction where computers can become a (semi-autonomous) partner in the human-human interaction. As more researchers get involved in these topics it becomes clear that our current understanding of user concerns is too much derived from the world of work and efficiency and it should be complemented with understanding of social partner concerns, including the different roles of humor.

Recently, embodied conversational agents (ECAs) have become a well-established research area. Embodied agents are agents that are visible in the interface as animated cartoon characters or resembling human beings. Sometimes they just consist of an animated talking face, displaying facial expressions and, when using speech synthesis, having lip synchronization. These agents are used to inform and explain or even to demonstrate products or sequences of activities in educational, e-commerce or recreational settings (Fig. 1). Experiments have shown that ECAs can in- 
crease the motivation of a student or a user interacting with the system. Current research deals with improving intelligent behavior of these ECAs, but also with adding emotional behavior and personality in order to make them more believable to the user and to induce trust. Event appraisal theories to model emotions are being used in this field. For example, in the Carmen project [6] event appraisal is used to recognize and process feelings of guilt and anger in a setting where an embodied conversational agent talks with a mother of children with leukemia.

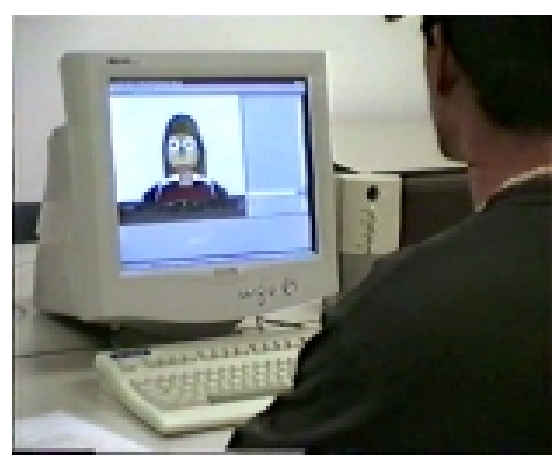

Fig. 1: User interacting with an embodied

agent. When should the agent smile?
mor, e.g. a humorous remark that is adequate in the context of the dialogue or aims at playing a social and/or regulating role in the interaction.

\section{HUMOR THEORY AND HCI}

In order to generate and comprehend humor we need computational theories of humor. There are theories of humor and some of them are oriented towards formal models that indeed can be used $[4,8,11]$, but at this moment certainly not in every domain, not for every application and not for every type of interaction. To identify which theories are best suited for human-computer interaction and how they should be developed further in order to become really useful is a challenge which we think that it is time to meet.

One possible way of proceeding is to review existing theories and observations concerning the appraisal of (humorous) situations (available as events, in conversation, in verbal descriptions or stories) in terms of possible agent models that include explicit modules for beliefs, desires, intentions and emotions. For example, it would be worthwhile to investigate how such a model can include reasoning mechanisms about situations where there is the feeling that the situation is normal, while at the same time there is a violation of a commitment of the agent about how things ought to be. With this view in mind it is useful to look at attempts to define degrees of incongruity [3], attempts to define humor in terms of violations of Grice's conversational maxims [1], proposals to define and explain humor or laughter in terms of perceptual, affective, and response patterns, but also 'measures' from questionnaires that are used to measure the propensity to laugh in a variety of verbally described situations.

\section{GOAL OF THE WORKSHOP}

This workshop aims to:

- $\quad$ provide a forum to discuss the role of humor in humancomputer interaction; can humor in the interface have similar effects as in interpersonal interactions and when do we want these effects?

- discuss a research agenda, including the identification of relevant (computational) humor theories, shortcomings and necessary development of theories; this will be done with future interface applications in mind.

In this workshop we hope to attract researchers from a wide range of disciplines, including Human-Computer Interaction, Computer Science, Computational Linguistics, Artificial Intelligence, Psychology and Social Science.

\section{REFERENCES}

1. S. Attardo. Violations of conversational maxims and cooperation. J. of Pragmatics 19 (1), 1993, 537-558.

2. A. Cann et al. On the role of humor appreciation in interpersonal attraction: It's no joking matter. Humor. J. of Humor Research (10) 1, 1997, 77-89.

3. L. Deckers. On the validity of a weight-judging paradigm for the study of humor. Humor. J. of Humor Research (6) 1, 2001, 43-56.

4. J. Hulstijn \& A. Nijholt (eds.). Computational Humor. IWCH'96, University of Twente, 1996.

5. J. Klein, Y. Moon, R.W. Picard. This computer responds to user frustration: Theory, design, and results. Interacting with Computers 14, Nr. 2, 2002, 119-140.

6. S.C. Marsella et al. Interactive pedagogical drama. In: Proc. 4th Intern. Conf. on Autonomous Agents 2000, ACM Press, 301-308.

7. J. Morkes, H.K. Kernal, and C. Nass. Humor in taskoriented computer-mediated communication and humancomputer interaction. In: Proc. CHI 98, 1998.

8. M.P. Mulder \& A. Nijholt. Humour research: State of the art. Deliverable IST Programme on Future and Emerging Technologies. University of Twente, 2001.

9. A. Nijholt. Embodied Agents: A New Impetus to Humor Research. In: [11], 101-111.

10.B. Reeves \& C. Nass. The Media Equation: how people treat computers, televisions and new media like real people and places. Cambridge University Press, 1996.

11. O. Stock, C. Strapparava \& A. Nijholt (eds.). Proc. The April Fools' Day Workshop on Computational Humour, ITC-irst, Trento, 2002. 